\title{
Editorial
}

\section{Computational Intelligence in Civil and Hydraulic Engineering}

\author{
Shengyong Chen, ${ }^{1}$ Fei Kang, ${ }^{2}$ Siamak Talatahari, ${ }^{3}$ Sunghwan Kim, ${ }^{4}$ and Dogan Aydin ${ }^{5}$ \\ ${ }^{1}$ College of Computer Science \& Technology, Zhejiang University of Technology, Hangzhou 310023, China \\ ${ }^{2}$ Faculty of Infrastructure Engineering, Dalian University of Technology, Dalian 116024, China \\ ${ }^{3}$ Marand Faculty of Engineering, University of Tabriz, Tabriz 51368, Iran \\ ${ }^{4}$ Department of Civil, Construction, and Environmental Engineering, Iowa State University, IA 50011, USA \\ ${ }^{5}$ Computer Engineering Department, Dumlupinar University, 43100 Kutahya, Turkey \\ Correspondence should be addressed to Shengyong Chen; sy@ieee.org
}

Received 30 April 2013; Accepted 30 April 2013

Copyright (C) 2013 Shengyong Chen et al. This is an open access article distributed under the Creative Commons Attribution License, which permits unrestricted use, distribution, and reproduction in any medium, provided the original work is properly cited.

Computational intelligence $(\mathrm{CI})$ is a set of nature-inspired computational methodologies and approaches that address complex problems of the real-world applications to which traditional methodologies and approaches are ineffective or infeasible. CI methods and techniques, including neural networks, evolutionary computation, and fuzzy logic systems, have rapidly evolved over the last decades, and they have already been applied in various fields of civil and hydraulic engineering. The focus of this special issue is the mathematical analysis and real-world application in civil and hydraulic engineering of computational intelligence. It is mostly interested in the related new development of both theoretical study and practical implementation, either with modeling, prediction, and optimization in geotechnical engineering, transportation engineering or structural design, diagnostics, and health monitoring.

The papers selected for this special issue represent a good panel in recent challenges. The topics of the research papers and review papers are connected with the computational intelligence methods and their application in civil and hydraulic infrastructure engineering, for example, structure optimization using evolutionary algorithms, damage detection using swarm intelligence, civil infrastructure performance prediction using neural networks, numerical simulation of intelligence algorithms, and civil infrastructures.

This special issue contains 29 papers. In the category of civil infrastructure engineering optimization design, $\mathrm{M}$. Polat Saka and Z. Woo Geem present an extensive review of mathematical and metaheuristic applications in design optimization of steel frame structures. X. Jun et al. present a nonlinear optimization technique for tunnel construction based on DE and LSSVM. H. Li et al. present a particle swarm optimization algorithm coupled with finite element limit equilibrium method for geotechnical practices. J.-T. Qu and $\mathrm{H}$. Li present a report on optimal placement of passive energy dissipation devices by genetic algorithms. X. Wang et al. present a method for three-dimensional target tracking in underwater wireless sensor networks using combination of interacting multiple models with the particle filter. N. Noilublao and S. Bureerat present a report on simultaneous topology, shape, and sizing optimisation of plane trusses with adaptive ground finite elements using MOEAs. S. Talatahari et al. present accelerated particle swarm optimization algorithm for optimal design of frame structures. S. Talatahari et al. present a report on optimum design of gravity retaining walls using charged system search algorithm. D. Wang et al. present a tangible programming tool with graphical blocks, that is, E-Block. Y.-S. Juang et al. present a report on histogram modification and wavelet transform for highperformance watermarking.

In the category of neural network in civil engineering, F.-K. Huang et al. present a report on rainfall reliability evaluation using artificial neural networks for stability of municipal solid waste landfills on a slope. X. Wang et al. present a report on inverse parametric analysis of seismic permanent deformation for earth-rockfill dams using artificial neural networks. R. K. Moghadas et al. present a report 
on the prediction of optimal design and deflection of space structures using neural networks. P. Lu et al. give a review on artificial intelligence in civil engineering. Z. Xie et al. present a report on freshwater algal bloom prediction by a supporting vector machine in macau storage reservoirs. I. Lou and Y. Zhao present a sludge bulking prediction method using principle component regression and artificial neural network.

In the category of numerical simulation of infrastructure engineering problems, F. Kang et al. present a structural damage detection method using combined data with particle swarm optimization. X.-W. Ye et al. present an intelligent risk assessment for dewatering of metro-tunnel deep excavations. Y.-J. Zheng et al. present a report on adaptive parameters for a modified comprehensive learning particle swarm optimizer. $\mathrm{H}$. Lin et al. present a simple generation technique of complex geotechnical computational model. X. Tang et al. present a report on factors influencing quasistatic modeling of deformation and failure in rock-like solids by the smoothed particle hydrodynamics method. W.-J. Niu et al. present a report on settlement analysis of a confined sand aquifer overlain by a clay layer due to single-well pumping. P. Lu et al. present a report on analysis of a T-frame bridge. S. Huaizhi et al. present a report on statistical analysis and calculation model of flexibility coefficient of low-and medium-sized arch dam. M. Carlini et al. present a report on down-hole heat exchangers: modeling of a low-enthalpy geothermal system for district heating. W. Wang et al. present a method for real-time simulation of fluid scenes by smoothed particle hydrodynamics and marching cubes. A. Kiliçman et al. present a report on numerical solution of nonlinear Volterra integral equations system using Simpson's 3/8 rule. Y. Liu et al. present a report on investment decision support for engineering projects based on risk correlation analysis. G. Wang et al. present a Lévy-Flight krill herd algorithm for solving optimization tasks within limited computing time.

Of 72 submissions, 29 papers are selected in this special issue. Of course, the topics and papers are not an exhaustive representation of the area of computational intelligence in civil infrastructure engineering. It can be seen that although some solutions and models become available, most problems remain open and research is highly active in this field. In the near future, we expect more contributions that will address all of the key aspects previously mentioned. Nonetheless, the special issue represents the recent concerns in the community and we have the pleasure of sharing them with the readers.

\title{
Acknowledgment
}

We would like to thank the authors for their excellent contributions and the reviewers in helping improving the papers.

\author{
Shengyong Chen \\ Fei Kang \\ Siamak Talatahari \\ Sunghwan Kim \\ Dogan Aydin
}




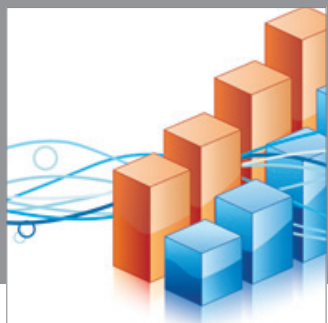

Advances in

Operations Research

mansans

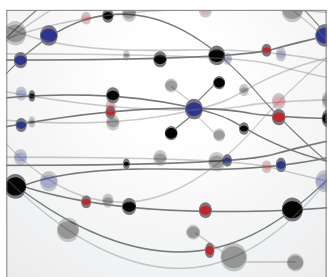

The Scientific World Journal
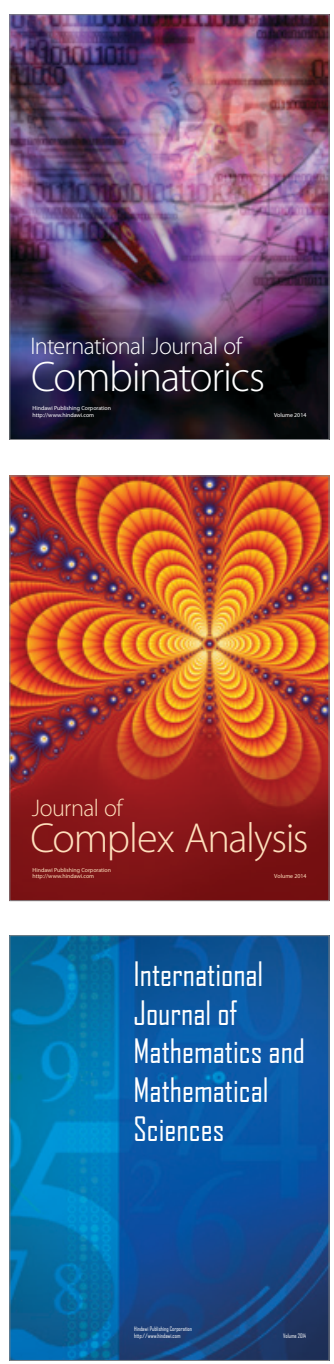
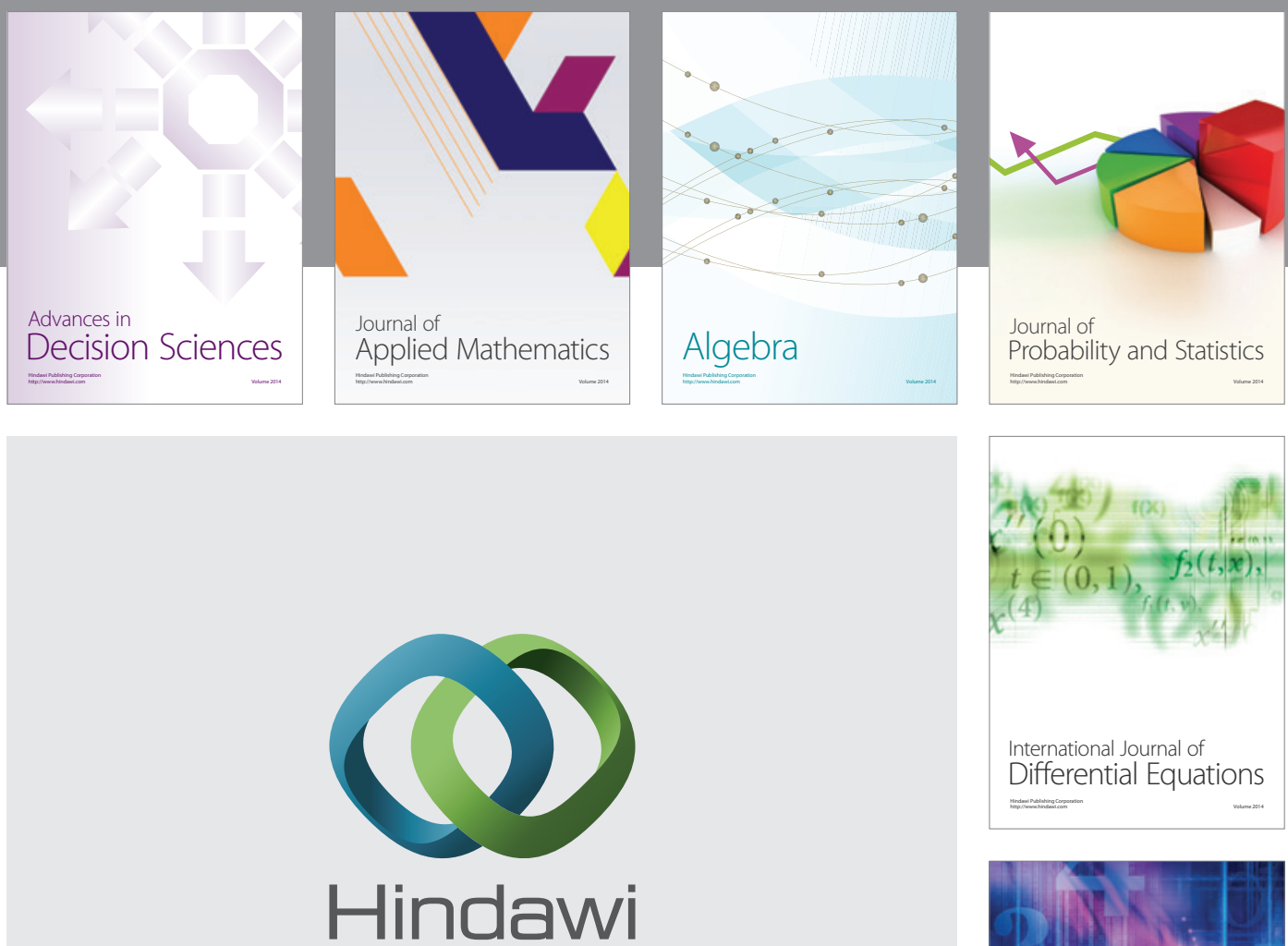

Submit your manuscripts at http://www.hindawi.com
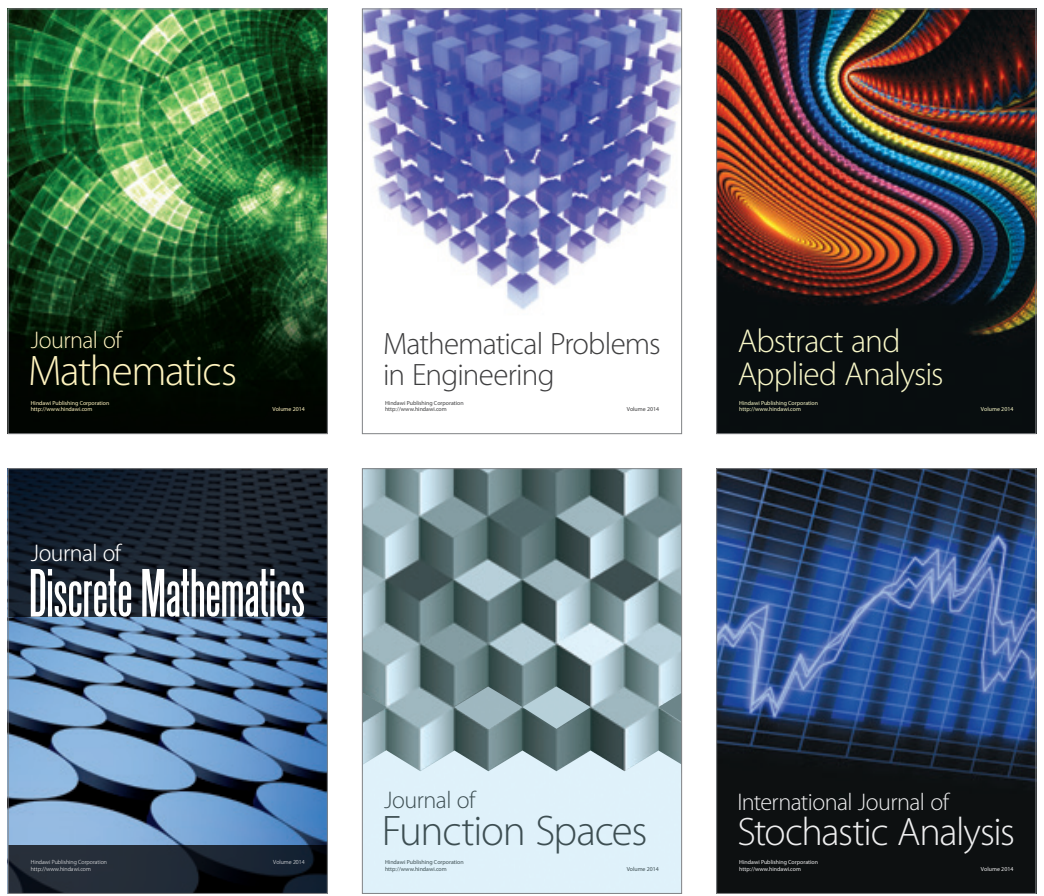

Journal of

Function Spaces

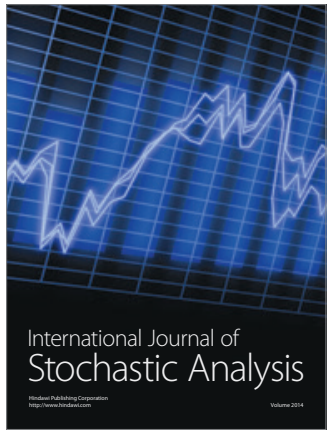

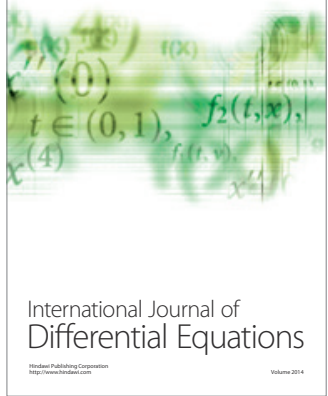
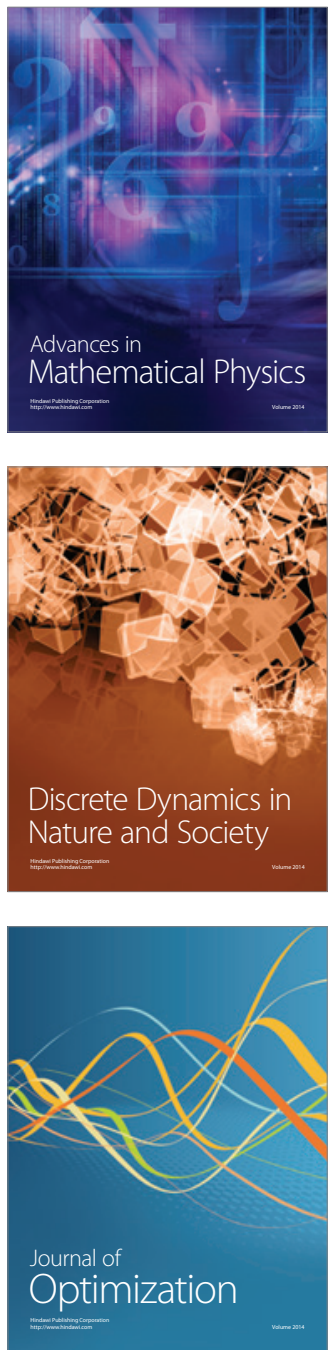